Letters to the Editor

\section{Safe Disposal of Air Discharged from Centrifuges}

We wish to draw attention to the introduction in some laboratories of a potentially unsafe method of disposing of centrifuge effluent air through safety cabinets with an extractor fan, without having first ensured that the volume of air discharged from the centrifuge does not exceed the exhaust capacity of the safety cabinet.

All centrifuges circulate air when in use and if the outside of the load is contaminated then the effluent air may well be so. In a centrifuge of recent introduction (BTL Intermediate/Analmatic) exhaust air leaves the machine by means of a $2 \frac{1}{8}$ in. internal diameter metal tube at its rear. We understand that in some laboratories this tube has been extended by means of pipework so as to terminate inside a safety cabinet, with the intention that the cabinet fan can discharge the exhaust air from the centrifuge through filters and thence to the external atmosphere. Such an arrangement gives the user a false sense of security: it can do little else since these centrifuges discharge 220 cubic feet of air per minute when rotating at maximum speed, whereas a safety cabinet, such as the PHLS protective cabinet 1970 model, when correctly installed and maintained to provide the air flow through its opening of 100 linear feet per minute (HM $70(60)$ ), is discharging only 170 cubic feet of air per minute through its exhaust ducting. By no means do all commercially available cabinets attain even this figure.

We prevent contamination of the effluent air by enclosing specimens in sealed plastic bags before centrifugation and we will continue to do so until such time as sealed buckets become generally available.

C. A. MORRIS

P. H. EVERALL

Public Health Laboratory, Shrewsbury

\section{Cryostat Sections of Undemineralized Bone}

We should like to comment on the interesting paper by Pearse and Gardner(1972). Although the need for cutting sections of undecalcified bone has been well recognized, the methods previously available involved either grinding or fixation and embedding in a very hard matrix, such as methacrylate (Sissons, 1968). The recent study by Raina (1972) has shown the value of the latter technique in the investigation of normal osteoid tissue. Pearse and Gardner (1972) drew attention to the possible importance of cutting sections of unfixed, undemineralized bone. For this purpose they designed and constructed a special bone cryostat. Even though they found difficulty in cutting serial sections with this instrument, it represented a considerable advance because, as they explained, 'it was not possible to cut adequate sections of fully mineralized bone on any cryostat microtome available in the United KingJom'.

In view of the advantages of cryostat sections for rapid diagnostic purposes and because such sections permit histochemical studies on bone physiology and pathology, we felt that our more recent experience with conventional and readily available apparatus might be of interest.

We are using the Jung rotary microtome 1130 in the normal Bright's Jung cryostat, FS/JS, as supplied by Bright Instrument Co Ltd. The temperature of the cryostat has been kept at $-25^{\circ} \mathrm{C}$. Hemisections of the bisected head of human radius have been cut with the normal Jung 'wedge profile' knife; a tungsten-tipped microtome knife has bzen found to be useful for cutting intact rat and mouse knee joints. With the former we have found that sections cut most readily if the knife is set so that its outward-facing edge subtends an angle of $50^{\circ}$ to the horizontal at its tip. Serial sections of all these tissues have been cut; sections of $5 \mu$ up to $20 \mu$ have been readily obtained.

Certain differences between this technique and that of Pearse and Gardner may be noted: (1) there is no need for the costly geared direct drive mechanism; it is not necessary to cut the sections slowly; (2) the angle of the block, the knife, and the guide-plate can be varied at will so that normal good cryostat microtomy technique can be used.

In our experience there is no special difficulty in cutting sections of chilled, undemineralized human bone or animal joints provided that the normal skills, required for any cryostat sectioning, are used. One major and unusual problem is that the sections tend to stick on the knife and to leave the bone on the knife when the section is transferred to a glass slide. This can be overcome by lightly coating the knife with a fine film of low temperature oil (as supplied by Bright Instrument Co Ltd).
The sections obtained by these means, with conventional and readily obtinable apparatus, have all the advantages described by Pearse and Gardner (192). The ease with which such sections caffbe obtained with commercially avaißble apparatus indicates that this techneque may well be of value in rapid diagnogic histology.

$$
\text { LUCILLE BITENSTKY }
$$$$
\text { J. JOHNSTONE }
$$

J. CHA $\overrightarrow{X E N}$

Division of Cellular Biology, Kennedy Institute of Rheumatology, Bute Gardens, Hammershith London W6 $78 \mathrm{~W}$

\section{References}

Pearse, A. D., and Gardner, D. L. (1972). $\overrightarrow{\text { Pdep- }}$ aration of unfixed undemineralized wone sections: the Bright bone cryostat. J.olin. Path., 25, 26-29.

Raina, V. (1972). Normal osteoid tissue. J. -tin. Path., 25, 229-232.

Sissons, H. A. (1968). Preparation of unde fied bone sections. Ass. clin. Path. B?adsheets, 62.

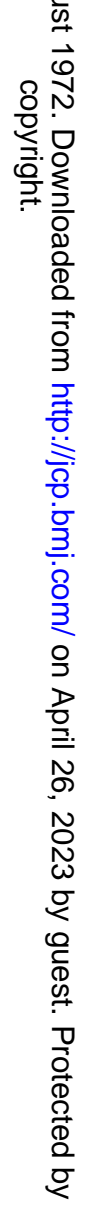

